\title{
Impacts of medroxyprogesterone acetate on oocytes and embryos: matched case-control study in women with stage III-IV ovarian endometriosis undergoing controlled ovarian hyperstimulation for in vitro fertilization
}

\author{
Haiyan Guo, Hongyuan Gao, Jianghui Li, Yanyan Cong, Qiuju Chen, Yun Wang, Qianqian Zhu, \\ Qifeng Lyu, Ling Wu, Weiran Chai, Yanping Kuang \\ Department of Assisted Reproduction, Shanghai Ninth People's Hospital, Shanghai Jiaotong University School of Medicine, Center for Specialty \\ Strategy Research of Shanghai Jiao Tong University China Hospital Development Institute, Shanghai 200011, China \\ Contributions: (I) Conception and design: Y Kuang, H Guo; (II) Administrative support: Y Kuang; (III) Provision of study materials or patients: H \\ Guo, H Guo, J Li, Y Cong, Q Chen, Y Wang, Q Zhu, Q Lyu, L Wu, W Chai; (IV) Collection and assembly of data: H Guo, H Guo, J Li, Y Cong, \\ Q Chen, Y Wang, Q Zhu, Q Lyu, L Wu, W Chai; (V) Data analysis and interpretation: H Guo; (VI) Manuscript writing: All authors; (VII) Final \\ approval of manuscript: All authors. \\ Correspondence to: Weiran Chai; Yanping Kuang. Department of Assisted Reproduction, Shanghai Ninth People's Hospital, Shanghai Jiaotong \\ University School of Medicine, Center for Specialty Strategy Research of Shanghai Jiao Tong University China Hospital Development Institute, \\ Shanghai 200011, China. Email: chaiwrcn@163.com; kuangyanp@126.com.
}

Background: This study investigated the effects of medroxyprogesterone acetate (MPA) on the oocytes and embryos in patients with advanced endometriosis who had a normal ovarian reserve and tubal infertility and received controlled ovarian hyperstimulation $(\mathrm{COH})$ and explored the characteristics and pregnancy outcomes in subsequent frozen-thawed embryo transfer (FET) cycles.

Methods: In this prospective controlled study, 150 advanced endometriosis patients involving 150 in vitro fertilisation/intracytoplasmic sperm injection (IVF/ICSI) cycles and 163 FET cycles and 150 agematched tubal infertility patients requiring $150 \mathrm{IVF} / \mathrm{ICSI}$ cycles and $115 \mathrm{FET}$ cycles were recruited. Patients with endometriosis were sub-grouped into surgery group ( $n=102)$ (they were diagnosed with ovarian endometriomas and underwent 102 IVF/ICSI and 115FET cycles) and aspiration group ( $\mathrm{n}=48$ ) [they had ovarian "chocolate" cysts $(>3 \mathrm{~cm})$ that were aspirated and underwent 48 IVF/ICSI and 74 FET cycles].

Results: Lower oocyte retrieval rate was noted in the endometriosis group than in the control group. Similar oocyte yield and peak estrogen $\left(\mathrm{E}_{2}\right)$ level were found in two groups. The rates of mature oocyte, fertilization, cleavage, high-quality embryo, viable embryo, cancellation, implantation, and clinical pregnancy were similar between two groups. A higher oocyte yield was observed in the EMS cyst group than in the surgery group.

Conclusions: The ovary response, oocytes, embryos and pregnancy outcome were not influenced by the advanced endometriosis and the use of MPA and also independent of endometrioma or cyst surgery.

Keywords: Endometriosis; medroxyprogesterone acetate (MPA); ovarian surgery; embryo transfer; pregnancy outcome

Submitted Nov 30, 2019. Accepted for publication Jan 18, 2020.

doi: 10.21037/atm.2020.02.15

View this article at: http://dx.doi.org/10.21037/atm.2020.02.15 


\section{Introduction}

Endometriosis is a well-known cause of infertility (1). The pathophysiological mechanisms by which endometriosis induces infertility remain unknown (2-4). Available molecular, histological and morphological studies have shown that endometriosis is harmful to the ovaries (5). Toxic content from an endometrioma may exert adverse effects such as increased oxidative stress and inflammation. It is probable that even mild endometriosis may adversely affect the oocyte development, embryogenesis and then implantation, resulting in the low fertility (6-10).

In recent years, the clinical outcome of ovarian endometrioma is a focus in the gynecological studies. It has been reported that surgical excision of these cysts may be harmful for the ovarian reserve $(11,12)$. Especially, the removal of bilateral endometriomas will significantly and adversely affect the IVF outcome $(12,13)$. Recently, studies have revealed that the responsiveness to ovarian hyperstimulation and the number of oocytes retrieved are significantly reduced in women with bilateral endometriomas compared with age-matched controls (14).

Progestins with good tolerability and low cost are the commonly used drugs for endometriomas and have been an economic alternative to surgery. MPA has been used as an alternative treatment to progestin for its unique advantages (15). Some studies have investigated the use of MPA in patients undergoing IVF (16-18). There is evidence showing that the embryos originating from MPA regimen in patients with advanced ovarian endometriosis have similar developmental potential as the short protocol (19). However, the responsiveness to hyperstimulation and the number of oocytes retrieved are still not unknown thoroughly from MPA protocol in patients with endometriomas $(>3 \mathrm{~cm})$ or undergoing cystectomy who have similar antral follicle count (AFC) as compared to tubal infertility during controlled ovarian hyperstimulation $(\mathrm{COH})$ in (in vitro fertilization) IVF treatment.

The influence of endometrioma and surgical intervention of endometriosis on the reproductive outcome of women who were treated with both MPA and hMG underwent IVF/ICSI are needed to be further confirmed. This prospective study was undertaken to (I) investigate the impact of endometrioma $(>3 \mathrm{~cm}$ ) on the IVF/ICSI outcomes and (II) explore the impact of surgery for endometrioma as compared to age-match tubal infertility controls with similar AFC.

\section{Methods}

\section{Patients}

This was a prospective case control study, and patients with endometriosis (cases) and those with tubal infertility (controls) were recruited from the Department of Assisted Reproduction of the Ninth People's Hospital of Shanghai Jiaotong University's School of Medicine between November 2015 and April 2016. The trial was registered in the Chinese Clinical Trial Registry (ChiCTRONC-15007399). This was performed according to the Declaration of Helsinki for Medical Research. All participants provided informed consents after counseling for infertility treatments and routine IVF procedures.

The patients with endometriosis were included according to the following criteria: (I) patients were aged 20-40 years, (II) the follicle stimulating hormone (FSH) was less than $10 \mathrm{IU} / \mathrm{L}$, (III) the AFC was 5-20, (IV) patients with diagnosed with advanced endometriosis on laparoscopy or laparotomy and had ovarian endometriomas that were treated surgically by laparoscopy or laparotomy before IVF or had ovarian endometriomas that were aspirated and identified as "chocolate" cysts $(>3 \mathrm{~cm}$ ) during ovulation monitoring or at the time of oocyte retrieval.

The tubal infertility patients who had the IVF/ICSI attempt were recruited in the same time period according to the following criteria: patients did not undergo prior ovarian surgery and did not have any ultrasonographic sign of endometriotic and/or non-endometriotic ovarian cysts at the time of the cycle; they were matched with the advanced endometriosis patients by age at the time of the cycle. The women with tubal factor infertility undergoing their first IVF/ICSI inclusion criteria were as follows: (I) aged 20 to 40 years; (II) FSH less than 10 IU/L; (III) AFC 5-20.

The exclusion criteria in the endometriosis group and tubal infertility group were as follows: (I) patients had polycystic ovarian syndrome (PCOS); (II) patients had hydrosalpinx; (III) patients had adenomyosis on the laparoscopy or laparotomy, ultrasonography displayed disordered myometrial echo, or magnetic resonance imaging (MRI) showed adenomyosis; (IV) patients had documented ovarian failure including basal FSH above $10 \mathrm{IU} / \mathrm{L}$. The cases and controls were age and AFC matched (1:1).

\section{Study design}

A total of 300 cycles in 300 patients were analyzed. Patients 
were divided into two groups: 150 cycles (EMS group, $\mathrm{n}=150$ ) were in women who were diagnosed with advanced endometriosis by laparoscopy or laparotomy and had ovarian endometriomas treated surgically before IVF or had ovarian endometriomas that were aspirated and identified as "chocolate" cysts $(>3 \mathrm{~cm})$ during ovulation monitoring or at the time of oocyte retrieval; 150 cycles (control group, $\mathrm{n}=150$ ) were in women undergoing IVF because of tubal factor infertility. Staged III-IV endometriosis was diagnosed according to the revised American Fertility Society (AFS) classification of endometriosis (20).

\section{Sample size}

This was a prospective non-inferiority trial. The sample size was estimated according to previous studies $(16,21)$. The primary end-point was the number of oocytes retrieved. The mean number of oocytes retrieved was assumed to be 10.0 , and that for non-infertility was $<2$.0. It was assuming that the number of normal ova obtained was similar between endometriosis women and tubal infertility patients. Thus, the required sample size was 130 for each group to achieve an $\alpha=0.05$ and a power of 0.8 (PS power and sample size calculations, version 2.1.30). Given the possibility of $10 \%$ dropouts, 150 women were included in each group.

\section{Ovarian stimulation and embryo culture}

Women undergoing IVF followed the MPA + hMG protocol that has been reported in detail previously (21). The final stage of oocyte maturation was triggered with decapeptyl $(0.1 \mathrm{mg})$ (Ferring International Center SA, Germany) and hCG (2,000 IU; Lizhu Pharmaceutical Trading Co, China) (21). The embryo quality (the number/ uniformity of blastomeres and the degree of fragmentation) was assessed (22). All follicles with a diameter larger than $10 \mathrm{~mm}$ were retrieved. Oocytes were fertilized conventionally or by intracytoplasmic sperm injection (ICSI). Embryos were graded in the same way on the third day. 1 or 2 good-quality embryos were transferred, and the procedure for frozen and thawed embryos was carried out the same as the study group.

\section{Transfer of cryopreserved-thawed embryos}

Endometrial preparation in frozen embryo transfer cycles was performed in natural cycles, mild stimulation cycles, or hormone therapy cycles (19).

\section{Data collection}

The primary outcome was the number of oocytes retrieved. The secondary outcomes included the duration and dosage of hMG, number of mature oocytes (MII oocyte) retrieved, high/low ovary response, cycle cancellation rate, and clinical pregnancy rate. Cycles were cancelled because of hyper- or hypo-ovarian response. The hyper-response was defined as the serum estradiol level greater than $4,000 \mathrm{pg} / \mathrm{mL}$ and/or more than 20 follicles identified on ultrasonography before HCG administration. The hypo-response was defined as no more than three follicles on ultrasonography during ovarian hyperstimulation.

\section{Statistical analysis}

Data underwent a normal distribution test; data with normal distribution are presented as mean \pm standard deviation (SD) and those with abnormal distribution as median (range or interquartile range). Data were analyzed using nonparametric analysis, Kruskal-Wallis test and Mann-Whitney $\mathrm{U}$ test when appropriate. The post hoc Bonferroni method was used for the comparison of hormones at different time points. The Mann-Whitney $U$-test was used for the data with abnormal distribution. A value of $\mathrm{P}<0.05$ was considered statistically significant. All data were analyzed using the Statistical Package for the Social Sciences for Windows (SPSS, ver. 16.0, SPSS Inc., Chicago, IL, USA).

\section{Results}

\section{Patients' characteristics}

Figure 1 shows the characteristics of patients included in this study. A total of 300 women were divided into EMS group and control group. Three hundred women completed oocyte retrieval cycles (EMS group: 150 patients, 150 cycles; control group: 150 patients, 150 cycles) and 248 women completed FET cycles (EMS group: 144 patients, 189 cycles; control group: 104 patients, 115 cycles). Oocytes were observed in all the patients. In 263 cycles (EMS group: 89.33\%; control group: 86\%), the highest quality embryos were suitable for cryopreservation, while 37 patients (EMS group: $n=16$; control group: $n=21$ ) were excluded because there were no high-quality embryos.

There were no significant differences in the age, BMI, $\mathrm{AFC}$, baseline FSH, LH, E2 and $\mathrm{P}$ between two groups. However, the duration of infertility was longer and the primary infertility rate was significantly higher in the EMS group than in the control group $(\mathrm{P}<0.05)$ (Table 1). 


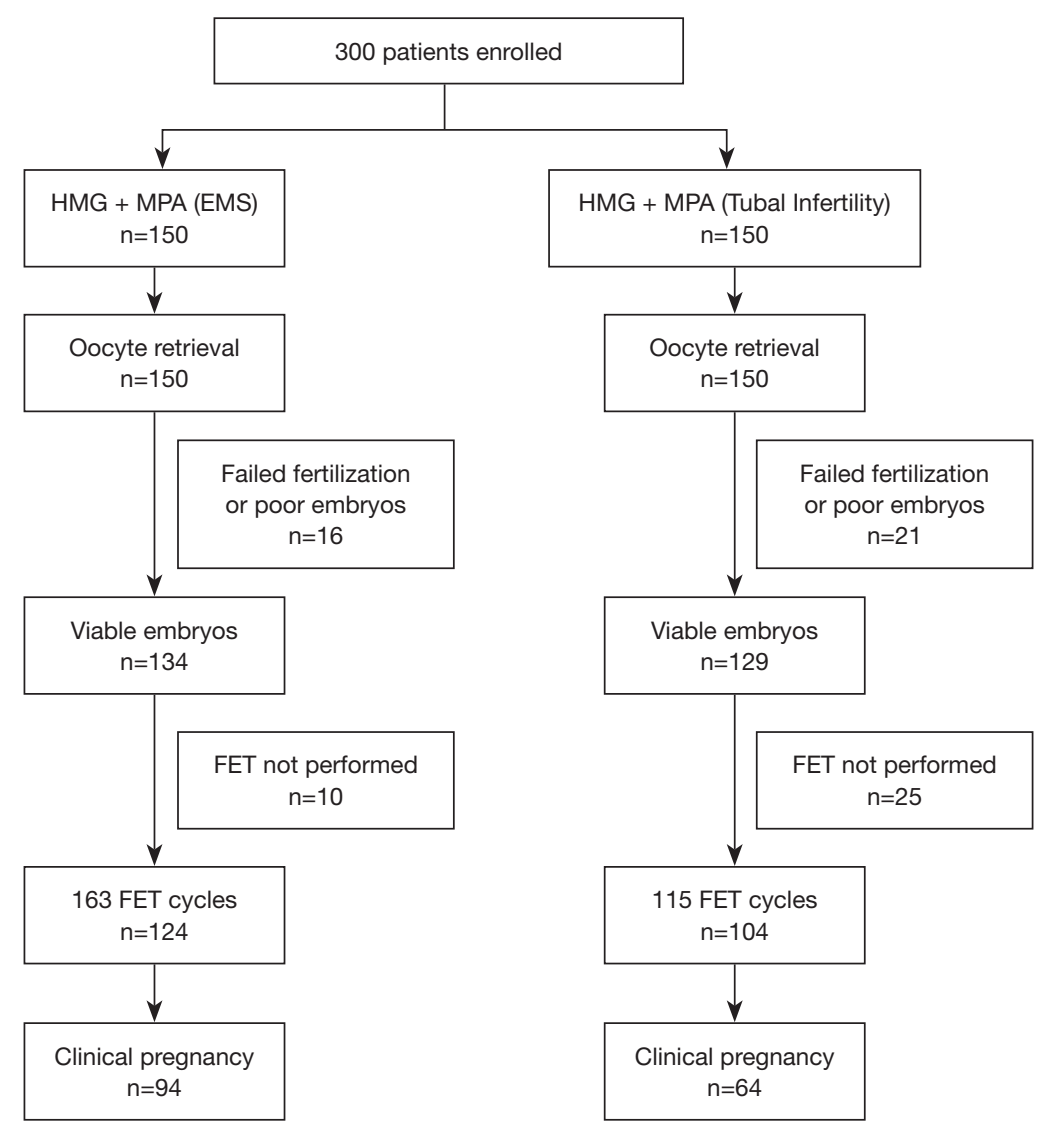

Figure 1 Consort flow diagram.

Table 1 The baseline characteristics and hormone profiles of women in two groups undergoing IVF/ICSI treatment

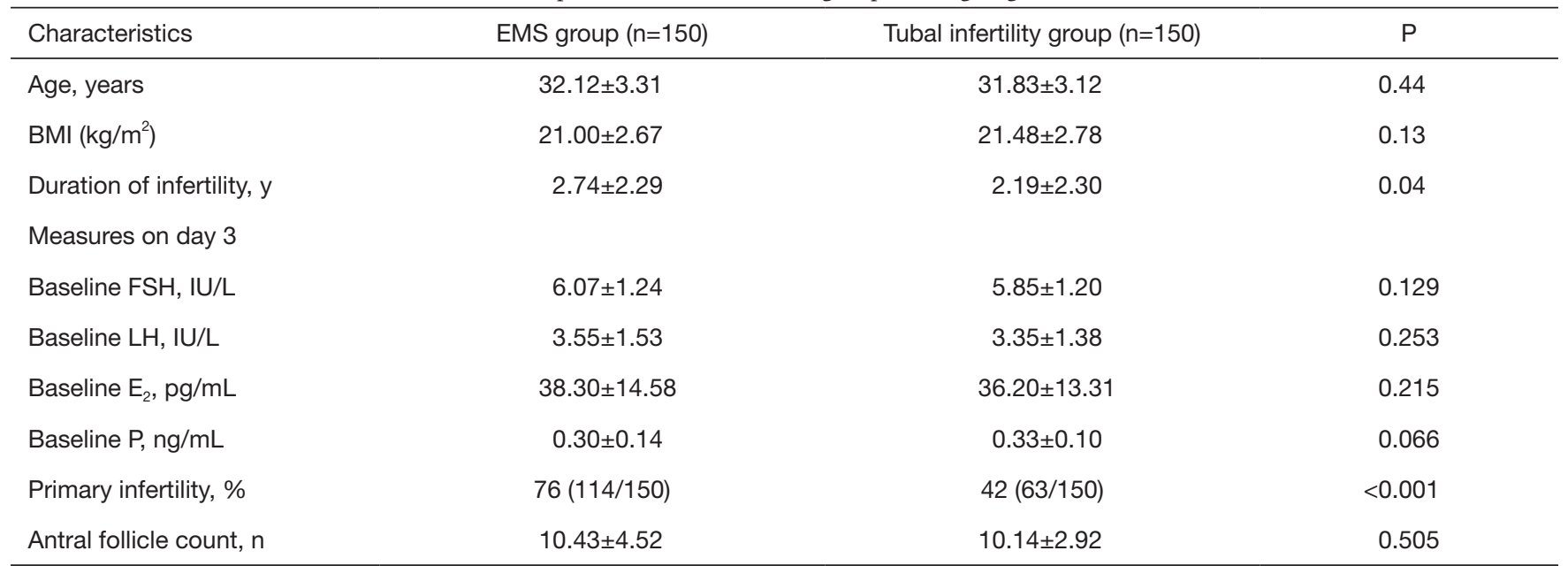

P: EMS group vs. control group. IVF, in vitro fertilization; ICSI, intracytoplasmic sperm injection; BMI, body mass index; EMS, endometriosis; FSH, follicle stimulating hormone; $\mathrm{LH}$, luteinizing hormone; $\mathrm{E}_{2}$, estrogen; $\mathrm{P}$, progesterone. 
Table 2 The baseline characteristics, cycle characteristics and pregnancy outcomes of frozen-thawed embryos in the surgery group and endometrioma group

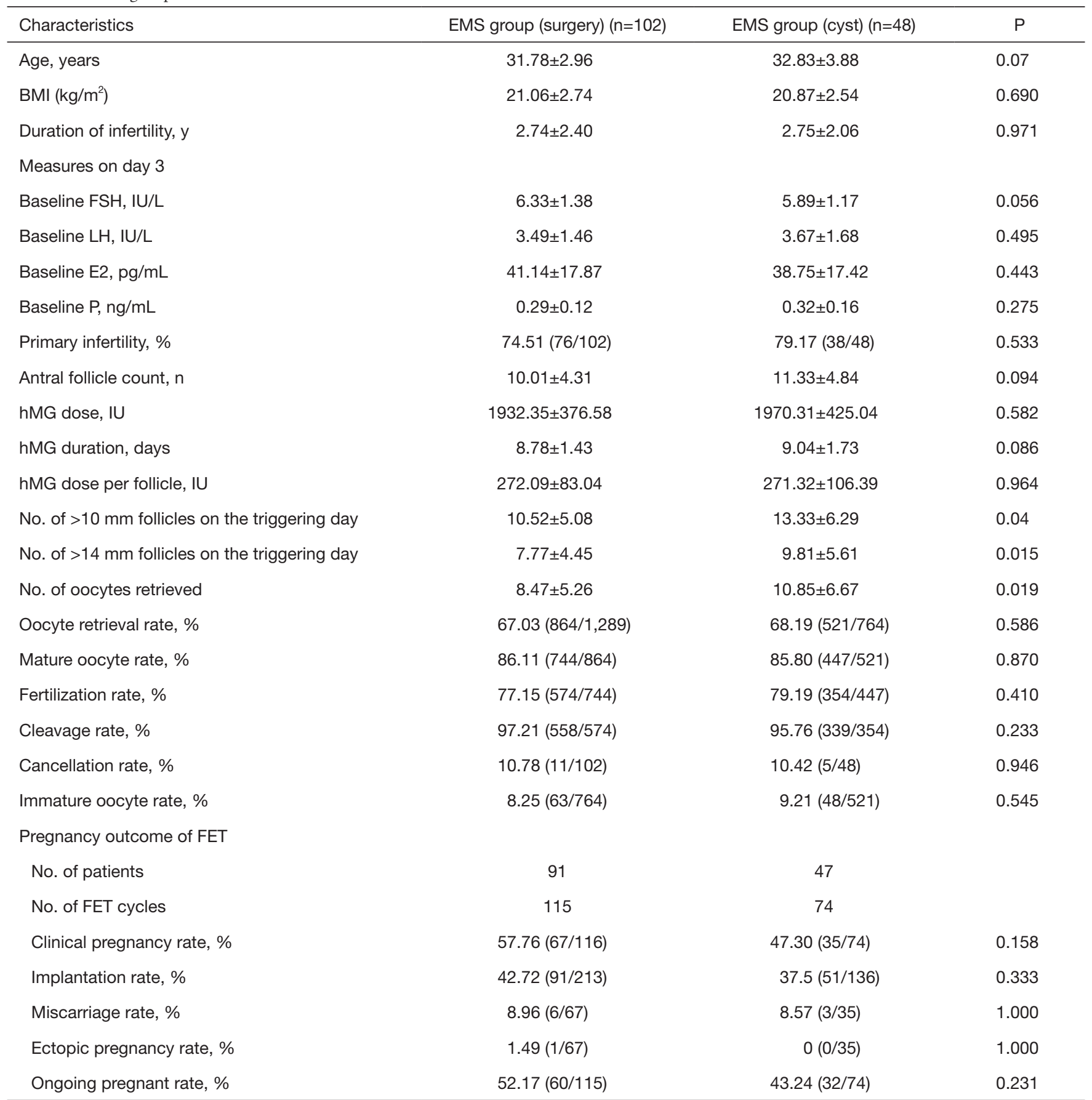

P: EMS surgery group vs. endometrioma group. IVF, in vitro fertilization; ICSI, intracytoplasmic sperm injection; FET, frozen-thawed embryo transfer. 

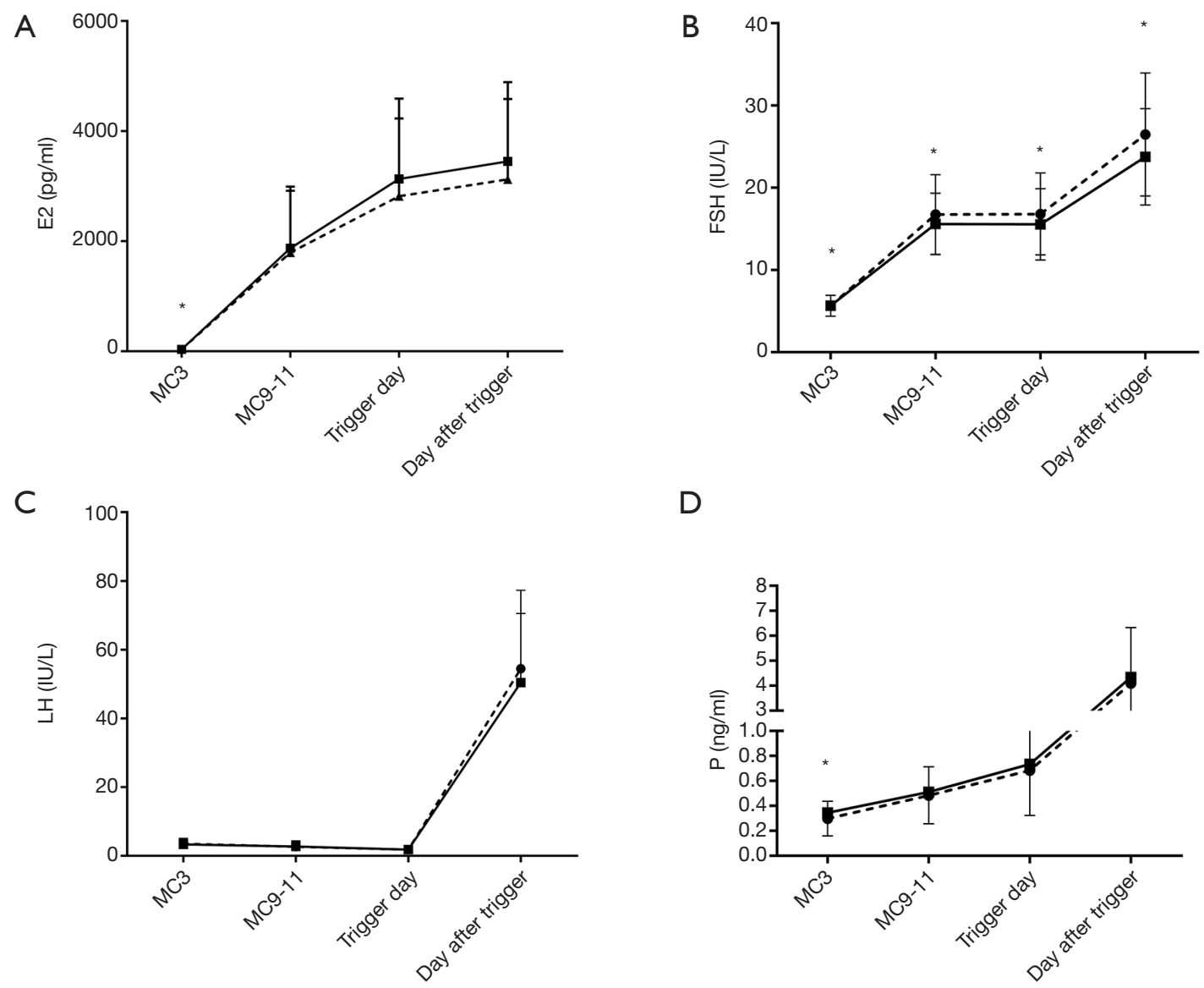

Figure 2 The hormone profiles of the medroxyprogesterone acetate (MPA) + human menopausal gonadotrophin (hMG) protocol in the two groups with trigger by gonadotropin-releasing hormone agonist (GnRH-a) or human chorionic gonadotrophin (hCG). The mean SD values show the temporal associations among circulating concentrations of follicle stimulating hormone (FSH), luteinizing hormone (LH), estrogen $\left(\mathrm{E}_{2}\right)$, and progesterone $(\mathrm{P})$. The dotted line shows the EMS group, the full line refers to the tubal infertility group. The asterisk $\left.{ }^{*}\right)$ represents $\mathrm{P}<0.05$ at the time point between EMS group vs. tubal infertility group. EMS, endometriosis.

Moreover, no significant differences were not observed in the baseline characteristics (age, BMI, duration of infertility, $\mathrm{AFC}$, previous failed FET cycles, primary infertility rate and baseline FSH, LH, E2 and P) between EMS surgery group and endometriomas group $(\mathrm{P}>0.05)$ (Table 2).

\section{Hormone profiles during treatment}

The blood levels of FSH, LH, E2, and P in the study groups are shown in Figure 2. FSH level increased significantly after hMG administration and remained stable during the ovarian stimulation. After triggering with decapeptyl and hCG, the average FSH level increased significantly (EMS group: $26.47 \pm 7.48 \mathrm{IU} / \mathrm{L}$, control group: $23.75 \pm 5.86 \mathrm{IU} / \mathrm{L}$, $\mathrm{P}<0.001)$. The $\mathrm{LH}$ level gradually decreased during ovarian stimulation, and the mean $\mathrm{LH}$ level on the triggering day was significantly lower than the baseline LH level (EMS group: $1.82 \pm 1.15$ vs. $3.55 \pm 1.53 \mathrm{IU} / \mathrm{L}$; control group: $1.85 \pm 1.12 v s$. $3.35 \pm 1.38 \mathrm{IU} / \mathrm{L}, \mathrm{P}<0.05)$ and then increased significantly (EMS group: $54.48 \pm 22.82 \mathrm{IU} / \mathrm{L}$, control group: $50.40 \pm$ $20.15 \mathrm{IU} / \mathrm{L}, \mathrm{P}<0.05)$ at $10 \mathrm{~h}$ after triggering. The $\mathrm{LH}$ level on the day after triggering was similar between EMS group and control group. Blood $\mathrm{E}_{2}$ level gradually increased, which was accompanied by the growth of follicles during ovarian stimulation; the $\mathrm{E}_{2}$ level increased significantly after triggering. The $\mathrm{E}_{2}$ level on the triggering day and the day after triggering were comparable between EMS group and control group $(2,817.23 \pm 1,411.57$ vs. $3,130.65 \pm 1,460.00 \mathrm{ng} / \mathrm{mL}, \mathrm{P}=0.072$; $3,125.19 \pm 1,458.51$ vs. $3,449.78 \pm 1,441.25 \mathrm{ng} / \mathrm{mL}, \mathrm{P}=0.066$ ). The $\mathrm{P}$ level was similar between two groups during ovarian 
Table 3 Cycle characteristics of women in two groups undergoing IVF/ICSI treatment

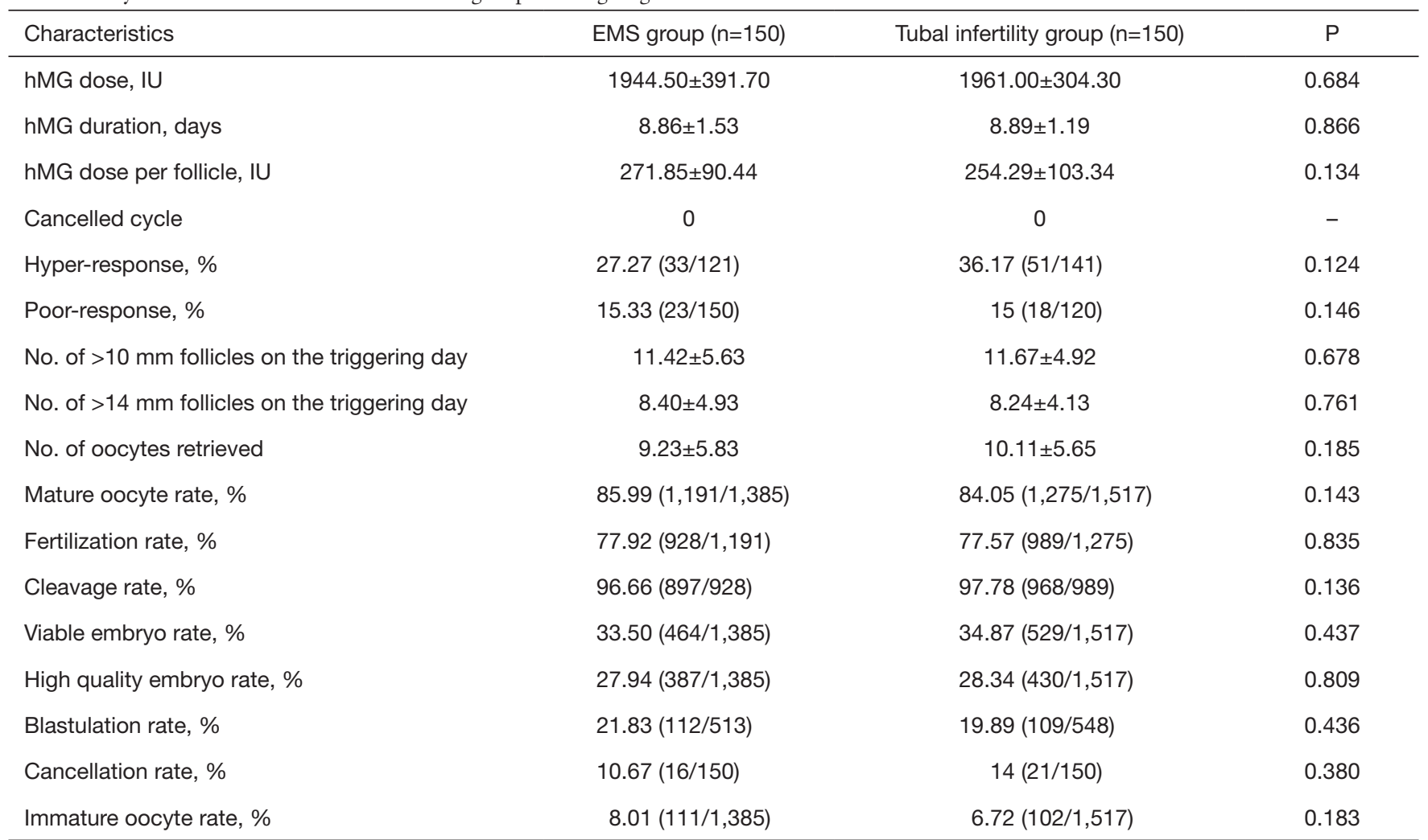

P: EMS group vs. control group. IVF, in vitro fertilization; ICSI, intracytoplasmic sperm injection; EMS, endometriosis; MPA, medroxyprogesterone acetate; HMG, human menopausal gonadotropin.

stimulation (Figure 2).

\section{Ovarian stimulation, follicle development, and oocyte performance}

The duration of stimulation and dose of hMG showed no significant differences between two groups (EMS group: $8.86 \pm 1.53$ days, control group: $8.89 \pm 1.19$ days; EMS group

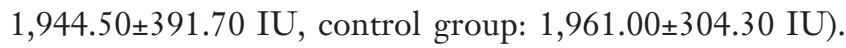
The number of follicles sized $>10 \mathrm{~mm}$ (EMS group: 11.42 \pm 5.63 ; control group: $11.67 \pm 4.92$ ) or $>14 \mathrm{~mm}$ (EMS group: $8.40 \pm 4.93$; control group: $8.24 \pm 4.13$ ) was similar between 2 groups. No significant differences were found in the oocyte maturation rate, number of high-quality embryos (D3; 6 grade I and II higher grade cells), or viable embryo rate of oocytes retrieved between groups $(\mathrm{P}>0.05)$. The number of oocytes retrieved was also similar between two groups (EMS group: $9.23 \pm 5.83$, control group: $10.11 \pm 5.65$; $\mathrm{P}>0.05)$. The rates of maturation $(85.99 \%$ vs. $84.05 \%)$, fertilization $(77.92 \%$ vs. $77.57 \%)$, cleavage $(96.66 \%$ vs.
97.78\%), viable embryos (33.50\% vs. 34.87\%), high-quality embryos (27.94\% vs. 28.34\%) were similar between EMS and control group (all $\mathrm{P}>0.05$ ). The cycle cancellation rate due to no viable embryos was not different between two groups (EMS group: 10.67\%, control group: 14\%; $>>0.05$ ). No patients experienced moderate or severe OHSS in the two groups (Table 3). In addition, the duration of stimulation, dose of hMG, number of follicles sized $>10-14 \mathrm{~mm}$ and number of oocytes retrieved were similar between two EMS groups. No significant differences were noted in the oocyte maturation rate, fertilization rate, cleavage rate and cancellation rate between two EMS groups $(\mathrm{P}>0.05)$ (Table 2).

\section{Pregnancy outcomes in FET cycles}

The FET pregnancy outcomes in two groups are shown in Table 4. A total of 228 women (EMS group: $n=124$; control group: $n=104$ ) in two groups completed a total of 278 FET cycles (EMS group: $n=163$; control group: $n=115$ ). The clinical pregnancy rate per transfer (EMS group: $57.67 \%$ vs. 
Table 4 Pregnancy outcomes after frozen-thawed embryo transfers in two groups

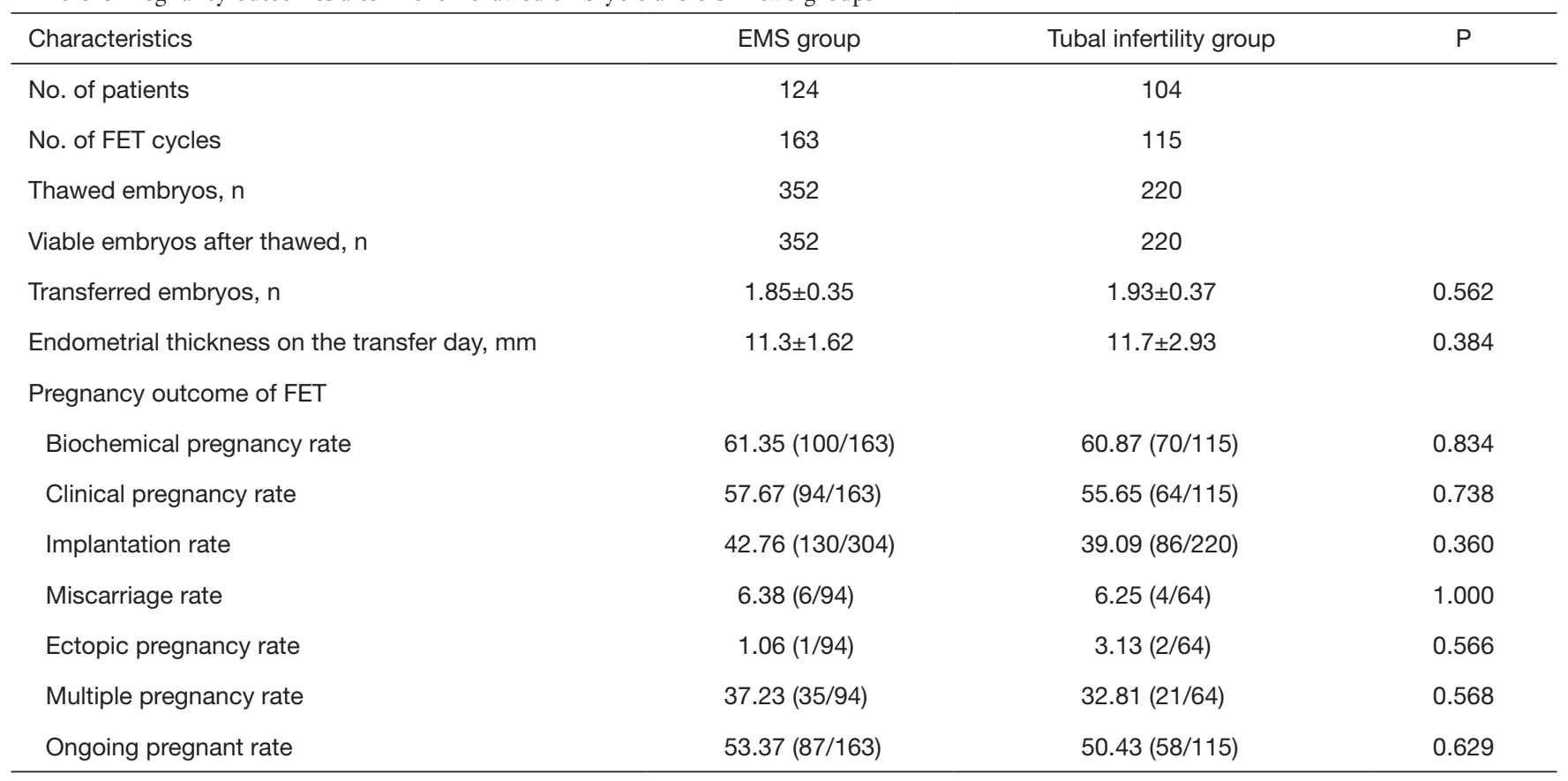

EMS, endometriosis; FET, frozen-thawed embryo transfer.

control group: $55.65 \%$ ) and implantation rate of embryos (42.76\% vs. 39.09\%) were similar between EMS group and control group. The proportion of multiple pregnancies (EMS group: $37.23 \%$ vs. control group: $32.81 \%$ ), miscarriage rate (EMS group: $6.38 \%$ vs. control group: $6.25 \%$ ) and ongoing pregnancy rate (EMS group: $53.37 \%$ vs. control group: $50.43 \%$ ) were similar between two groups $(\mathrm{P}>0.05)$ (Table 4). Moreover, no significant differences were found in the FET pregnancy outcomes between EMS surgery group and endometrioma group $(\mathrm{P}>0.05)$ (Table 2$)$.

\section{Discussion}

Our findings indicated that the number of retrieved oocytes and ovarian response to hyperstimulation were similar between endometriosis patients and tubal infertility patients. In addition, the number of mature oocytes, fertilization rate, proportion of high-quality embryos, and pregnancy outcomes were also comparable between endometriosis group and tubal infertility group. In the subgroup analysis, the number of oocytes retrieved reduced in the surgically treated endometriosis patients as compared to endometriomas patients without surgery. Moreover, the number of oocytes and embryo quality were not influenced by the endometriomas and surgery. This means that the quality of oocytes and embryos derived from severe endometriosis patients receiving the MPA regimen were not influenced by the surgery or endometriotic cysts. Studies investigating the morphology of oocytes and embryo development in women with and without endometriosis also reveal no differences in the implantation rate and pregnancy outcome (23-27), which are in line with our findings.

Endometrioma was not specifically examined in the presence of normal ovarian function in available studies. The expansion of results from most previous studies is limited by the methodology, sample size and accurate diagnosis $(28,29)$. In order to resolve these controversies, the present prospective study was designed to assess the pregnancy outcomes with normal ovary reserve, in which controls matched in age and AFC were recruited. Recent studies have paid attention to the impact of surgical treatment for endometrioma on the ovarian reserve (30-32) and IVF/ICSI reproductive outcomes $(33,34)$. However, the possible adverse effects of the endometrioma and the surgery for endometrioma on the oocytes and embryos in women with normal ovarian reserve during IVF/ICSI have not been studied. Our findings indicate the surgical removal of the cysts or the cysts themselves might not damage the oocytes and embryos in patients with normal ovarian reserve. Furthermore, the oocytes and embryos had similar 
developmental potential between patients receiving MPA protocol and tubal infertility patients. Therefore, based on our findings, the MPA and hMG protocol can be used without affecting the competent oocyte/embryo production in advanced endometriosis patient. The peak $\mathrm{E}_{2}$ level was comparable between control group and EMS group, which is not consistent with previous findings (35). This may be attributed to the normal ovary reserve in our study which is different from the inclusion criteria in previous studies.

Endometriosis is a disease related to the immune system dysfunction and inflammation, and anti-inflammatory and immunomodulatory treatments have been employed in the clinical treatment of endometriosis. Progestogens has been used in the treatment of endometriosis, which may be related to the improvement of granulosa cells and the microenvironment for the development of oocytes and embryos, which in return improves pregnancy outcomes $(36,37)$.

Compared with women undergoing surgery for endometrioma, women with surgery for cysts had a similar implantation rate and clinical pregnancy rate, although a smaller mean number of oocytes retrieved was noted after endometrioma surgery. In the IVF procedure, the overall cancellation rate was $10.67 \%$ in the EMS group and 14\% in the control group. The criterion for cancellation was no good-quality embryos. Our results showed endometriosis did not affect the embryo quality and the pregnancy related parameters (fertilization rate, embryo quality, implantation rate, pregnancy rate, and live birth rate), which was independent of active ovarian endometrioma or surgery treatment. Many patients have low ovarian reserve due to the operation of severe endometriomas, which leads to ovarian failure in some cases. Therefore, some patients with endometriomas have not been punctured due to very low possibility of malignancy excluded by B-ultrasound or MRI. The large ovarian cyst will affect the growth of follicles and oocytes retrieval during IVF, so ovarian cyst was punctured before oocytes retrieval. This choice may result in a bias in the findings. However, our results also revealed that endometriosis, even after laparoscopic treatment, affected the number of oocytes retrieved. In addition, following laparoscopic treatment, an ovarian endometrioma had no additional negative effects on the oocyte development. However, there is recent evidence showing that AFC is not a good option in evaluating ovarian reserve of endometriosis patients. Endometriomas, local inflammation, fibrosis, adhesions due to endometriosis and prior surgery may impair the visualization of follicles. AMH may serve as a better test for ovarian reserve. AMH was not counted, which may be a limit of the study. We will add this index in the follow-up study. Thus, more studies are needed to elucidate this issue and to confirm our findings.

\section{Acknowledgments}

Funding: This study was supported partially by the National Key Research and Development Program of China (No. 2018YFC1003000 to Y Kuang), the Natural Science Foundation of Shanghai (No. 81771574 to L Wu), and the Shanghai Municipal Commission of Health and Family Planning Foundation (No. 20174 Y0036 to L Wu).

\section{Footnote}

Conflicts of Interest: The authors have no conflicts of interest to declare.

Ethical Statement: The authors are accountable for all aspects of the work in ensuring that questions related to the accuracy or integrity of any part of the work are appropriately investigated and resolved. This was performed according to the Declaration of Helsinki for Medical Research. All participants provided informed consents after counseling for infertility treatments and routine IVF procedures.

Open Access Statement: This is an Open Access article distributed in accordance with the Creative Commons Attribution-NonCommercial-NoDerivs 4.0 International License (CC BY-NC-ND 4.0), which permits the noncommercial replication and distribution of the article with the strict proviso that no changes or edits are made and the original work is properly cited (including links to both the formal publication through the relevant DOI and the license). See: https://creativecommons.org/licenses/by-nc-nd/4.0/.

\section{References}

1. Macer ML, Taylor HS. Endometriosis and infertility: a review of the pathogenesis and treatment of endometriosisassociated infertility. Obstet Gynecol Clin North Am 2012;39:535-49.

2. Giudice LC. Clinical practice. Endometriosis. N Engl J Med 2010;362:2389-98.

3. Baldi A, Campioni M, Signorile PG. Endometriosis: pathogenesis, diagnosis, therapy and association with cancer (review). Oncol Rep 2008;19:843-6. 
4. Brown J, Farquhar C. Endometriosis: an overview of Cochrane Reviews. Cochrane Database Syst Rev 2014;(3):CD009590.

5. Sanchez AM, Vigano P, Somigliana E, et al. The distinguishing cellular and molecular features of the endometriotic ovarian cyst: from pathophysiology to the potential endometrioma-mediated damage to the ovary. Hum Reprod Update 2014;20:217-30.

6. Stilley JA, Birt JA, Sharpe-Timms KL. Cellular and molecular basis for endometriosis-associated infertility. Cell Tissue Res 2012;349:849-62.

7. Panidis DK, Matalliotakis IM. Subfertility associated with minimal to mild endometriosis. Main mechanisms. J Reprod Med 1998;43:1034-42.

8. Pauli SA, Session DR, Shang W, et al. Analysis of follicular fluid retinoids in women undergoing in vitro fertilization: retinoic acid influences embryo quality and is reduced in women with endometriosis. Reprod Sci 2013;20:1116-24.

9. Mahutte NG, Arici A. New advances in the understanding of endometriosis related infertility. J Reprod Immunol 2002;55:73-83.

10. Signorile PG, Baldi A. Endometriosis: new concepts in the pathogenesis. Int J Biochem Cell Biol 2010;42:778-80.

11. Garcia-Velasco JA, Somigliana E. Management of endometriomas in women requiring IVF: to touch or not to touch. Hum Reprod 2009;24:496-501.

12. Somigliana E, Arnoldi M, Benaglia L, et al. IVFICSI outcome in women operated on for bilateral endometriomas. Hum Reprod 2008;23:1526-30.

13. Tang Y, Chen SL, Chen X, et al. Ovarian damage after laparoscopic endometrioma excision might be related to the size of cyst. Fertil Steril 2013;100:464-9.

14. Benaglia L, Bermejo A, Somigliana E, et al. In vitro fertilization outcome in women with unoperated bilateral endometriomas. Fertil Steril 2013;99:1714-9.

15. Sitruk-Ware R, Nath A, Mishell DR, Jr. Contraception technology: past, present and future. Contraception 2013;87:319-30.

16. Kuang Y, Chen Q, Fu Y, et al. Medroxyprogesterone acetate is an effective oral alternative for preventing premature luteinizing hormone surges in women undergoing controlled ovarian hyperstimulation for in vitro fertilization. Fertil Steril 2015;104:62-70.e3.

17. Wang Y, Chen Q, Wang N, et al. Controlled Ovarian Stimulation Using Medroxyprogesterone Acetate and hMG in Patients With Polycystic Ovary Syndrome Treated for IVF: A Double-Blind Randomized Crossover Clinical Trial. Medicine (Baltimore) 2016;95:e2939.
18. Zhu X, Zhang X, Fu Y. Utrogestan as an effective oral alternative for preventing premature luteinizing hormone surges in women undergoing controlled ovarian hyperstimulation for in vitro fertilization. Medicine (Baltimore) 2015;94:e909.

19. Guo H, Wang Y, Chen Q, et al. Use of medroxyprogesterone acetate in women with ovarian endometriosis undergoing controlled ovarian hyperstimulation for in vitro fertilization. Sci Rep 2017;7:11927.

20. Revised American Fertility Society classification of endometriosis: 1985. Fertil Steril 1985;43:351-2.

21. Ren J, Sha A, Han D, et al. Does prolonged pituitary down-regulation with gonadotropin-releasing hormone agonist improve the live-birth rate in in vitro fertilization treatment? Fertil Steril 2014;102:75-81.

22. Cummins JM, Breen TM, Harrison KL, et al. A formula for scoring human embryo growth rates in in vitro fertilization: its value in predicting pregnancy and in comparison with visual estimates of embryo quality. J In Vitro Fert Embryo Transf 1986;3:284-95.

23. Yang X, Huang R, Cai M, et al. Endometriosis has no negative impact on outcomes of in vitro fertilisation in women with poor ovarian response. BJOG 2016;123 Suppl 3:76-81.

24. Suzuki T, Izumi S, Matsubayashi H, et al. Impact of ovarian endometrioma on oocytes and pregnancy outcome in in vitro fertilization. Fertil Steril 2005;83:908-13.

25. Reinblatt SL, Ishai L, Shehata F, et al. Effects of ovarian endometrioma on embryo quality. Fertil Steril 2011;95:2700-2.

26. Ashrafi M, Fakheri T, Kiani K, et al. Impact of the endometrioma on ovarian response and pregnancy rate in in vitro fertilization cycles. Int J Fertil Steril 2014;8:29-34.

27. Filippi F, Benaglia L, Paffoni A, et al. Ovarian endometriomas and oocyte quality: insights from in vitro fertilization cycles. Fertil Steril 2014;101:988-93.e1.

28. Vigano P, Corti L, Berlanda N. Beyond infertility: obstetrical and postpartum complications associated with endometriosis and adenomyosis. Fertil Steril 2015;104:802-12.

29. Leone Roberti Maggiore U, Ferrero S, Candiani M, et al. Bladder Endometriosis: A Systematic Review of Pathogenesis, Diagnosis, Treatment, Impact on Fertility, and Risk of Malignant Transformation. Eur Urol 2017;71:790-807.

30. Raffi F, Metwally M, Amer S. The impact of excision of ovarian endometrioma on ovarian reserve: a systematic 
review and meta-analysis. J Clin Endocrinol Metab 2012;97:3146-54.

31. Somigliana E, Berlanda N, Benaglia L, et al. Surgical excision of endometriomas and ovarian reserve: a systematic review on serum antimullerian hormone level modifications. Fertil Steril 2012;98:1531-8.

32. Muzii L, Di Tucci C, Di Feliciantonio M, et al. The effect of surgery for endometrioma on ovarian reserve evaluated by antral follicle count: a systematic review and metaanalysis. Hum Reprod 2014;29:2190-8.

33. Tsoumpou I, Kyrgiou M, Gelbaya TA, et al. The effect of surgical treatment for endometrioma on in vitro fertilization outcomes: a systematic review and metaanalysis. Fertil Steril 2009;92:75-87.

34. Benschop L, Farquhar C, van der Poel N, et al.

Cite this article as: Guo H, Gao H, Li J, Cong Y, Chen Q, Wang Y, Zhu Q, Lyu Q, Wu L, Chai W, Kuang Y. Impacts of medroxyprogesterone acetate on oocytes and embryos: matched case-control study in women with stage III-IV ovarian endometriosis undergoing controlled ovarian hyperstimulation for in vitro fertilization. Ann Transl Med 2020;8(6):377. doi: 10.21037/atm.2020.02.15
Interventions for women with endometrioma prior to assisted reproductive technology. Cochrane Database Syst Rev 2010CD008571.

35. Pop-Trajkovic S, Kopitovic V, Popovic J, et al. In vitro fertilization outcome in women with endometriosis \& previous ovarian surgery. Indian J Med Res 2014;140:387-91.

36. Pellicer A, Albert C, Mercader A, et al. The follicular and endocrine environment in women with endometriosis: local and systemic cytokine production. Fertil Steril 1998;70:425-31.

37. Pellicer A, Valbuena D, Bauset C, et al. The follicular endocrine environment in stimulated cycles of women with endometriosis: steroid levels and embryo quality. Fertil Steril 1998;69:1135-41. 